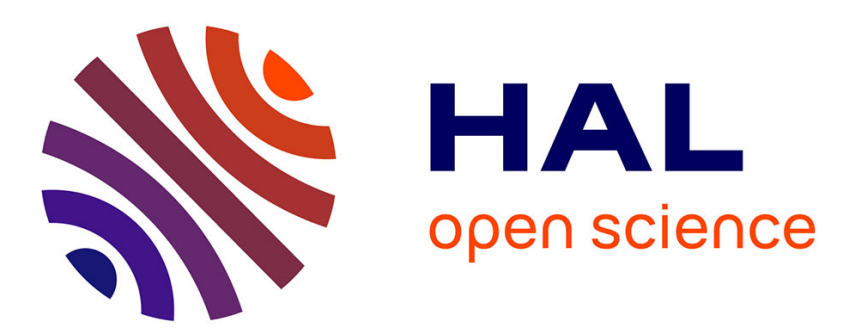

\title{
Photoexcitation et pompage optique dans un jet atomique de baryum
}

\author{
D. Aubert, A. Baldy, P. Baulaigue, H. Chantrel
}

\section{To cite this version:}

D. Aubert, A. Baldy, P. Baulaigue, H. Chantrel. Photoexcitation et pompage optique dans un jet atomique de baryum. Revue de Physique Appliquée, 1975, 10 (4), pp.187-194. 10.1051/rphysap:01975001004018700 . jpa-00243904

\section{HAL Id: jpa-00243904 https://hal.science/jpa-00243904}

Submitted on 1 Jan 1975

HAL is a multi-disciplinary open access archive for the deposit and dissemination of scientific research documents, whether they are published or not. The documents may come from teaching and research institutions in France or abroad, or from public or private research centers.
L'archive ouverte pluridisciplinaire HAL, est destinée au dépôt et à la diffusion de documents scientifiques de niveau recherche, publiés ou non, émanant des établissements d'enseignement et de recherche français ou étrangers, des laboratoires publics ou privés. 


\title{
PHOTOEXCITATION ET POMPAGE OPTIQUE DANS UN JET ATOMIQUE DE BARYUM
}

\author{
D. AUbERT, A. BALDY, P. BAUlaigue et H. CHANTREL \\ Laboratoire de Spectroscopie Atomique, Centre Scientifique de Saint-Jérome, \\ 13397 Marseille Cedex 4, France.
}

(Reçu le 17 octobre 1974, révisé le 3 février 1975, accepté le 19 février 1975)

\begin{abstract}
Résumé. - On décrit un dispositif permettant la photoexcitation d'un jet atomique de baryum sur toute son étendue. Cette photoexcitation est réalisée à l'aide d'un autre spectre du baryum émis en amont du jet par une décharge en cathode creuse produite à la sortie du four. Le spectre de fluorescence est étudié en détail, ainsi que l'influence de différents paramètres tels que la température du four, l'intensité du courant, la profondeur d'excitation. Un calcul montre comment il est possible de déterminer les coefficients d'Einstein, les forces d'oscillateur et les durées de vie atomiques à partir des mesures expérimentales.
\end{abstract}

\begin{abstract}
An apparatus allowing the photoexcitation of barium atomic beam over its whole surface is described. This photoexcitation is achieved by another barium spectrum emitted up stream of the beam by a cathodic out-flow produced at the exit oven. The fluorescence spectrum is studied in detail as well as the influence of different parameters, such as oven temperature, current intensity of the ouflow, and excitation depth. A calculation shows how to determine Einstein coefficient, oscillator strengths and atomic lifetimes from our experimental measurements.
\end{abstract}

Introduction. - Les techniques de photoexcitation sur des vapeurs atomiques sont largement utilisées depuis longtemps. Les études sur jets atomiques sont plus récentes et moins nombreuses (Odintsov) [6] (Ishii-Ohlendorf) [4].

Un jet atomique constitue un milieu à cinétique anisotrope où, à densité égale, les chocs sont très rares puisque tous les atomes se déplacent suivant des directions très voisines d'un même axe. Ceci permet de s'affranchir des transferts d'excitation par chocs interatomiques qui perturbent les expériences faites sur cuve.

La photoexcitation est produite par un faisceau lumineux superposé au jet atomique, édifié suivant le même axe avec sensiblement la même géométrie.

Dans ce qui suit, nous étudions ce nouveau pocédé d'excitation, l'appareillage réalisé et les premiers résultats obtenus sur un jet de baryum.

1. Etude sommaire du jet atomique. - Ce dispositif, maintenant classique, a été étudié par de nombreux auteurs (Stanley) [8] (Montixi) [5] (Zhiglinskii) [10].

Celui qui a été réalisé dans notre laboratoire comporte un four dont la température, régulée à $\pm 0,5^{\circ} \mathrm{C}$, est comprise entre $800^{\circ} \mathrm{C}$ et $900^{\circ} \mathrm{C}$. La vapeur de baryum sort du four par la fente $F_{1}$ de largeur $s=1 \mathrm{~mm}$ et de longueur $l=8 \mathrm{~mm}$ (Fig. 1). Une deuxième fente collimatrice $\mathrm{F}_{2}$ de mêmes dimensions est placée à la distance $h=30 \mathrm{~mm}$ au-dessus de la première. Elle sélectionne les atomes formant le jet. Le facteur de collimation du jet suivant sa largeur $s$ est $\mathrm{C}=h / s$.

Le débit du four est égal à $N_{1}$ at $/ \mathrm{s}=n_{1} \bar{v} \cdot s . l . / 4$ où $n_{1}=P / k T$ est le nombre d'atomes par unité de volume dans le four et $\bar{v}=\sqrt{8 \mathrm{kT} / \pi m}$ leur vitesse moyenne.

Le débit au niveau de la deuxième fente (donc à la base du jet) est $N_{2}$ at/s $=N_{1} \frac{s . l}{\pi h^{2}}$.

A $1000 \mathrm{~K}\left(P \sim 10^{-1}\right.$ torr $), N_{1}=10^{18}$ at $/ \mathrm{s}$ et $N_{2}=4 \times 10^{16}$ at $/ \mathrm{s}$. Ceci montre que $4 \%$ seulement des atomes émis par le four participent à la formation $\mathrm{du}$ jet.

Ces valeurs sont les plus grandes qu'on puisse atteindre avec les fentes employées. Pour des pressions de four plus élevées, le libre parcours moyen des atomes devient inférieur à la largeur des fentes, et le jet est détruit par la formation de turbulences au niveau de la fente collimatrice.

En tout point $\mathrm{A}$ du jet, la fente du four est vue sous un angle solide $\Omega$. Si $n_{0}$ est le nombre d'atomes par 


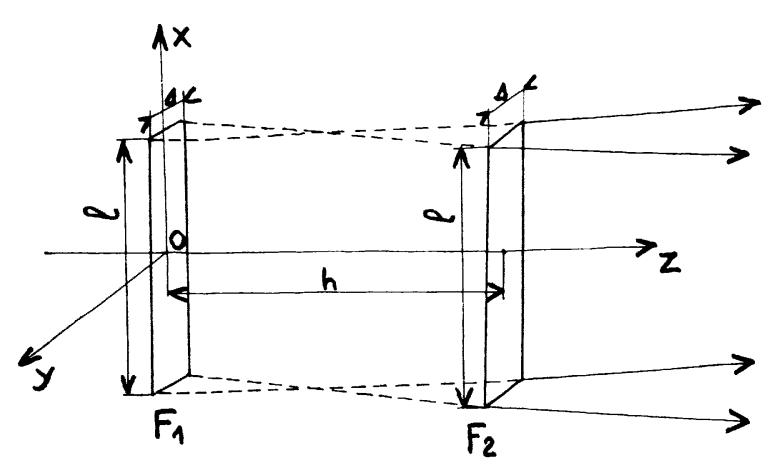

FIG. 1

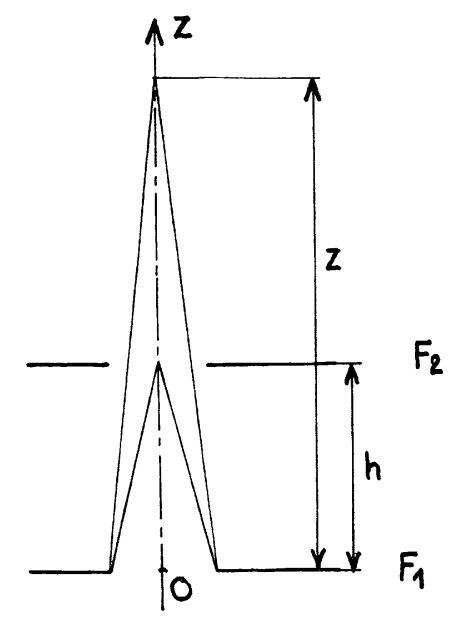

FIG. 2

FIG. 1 et 2. - Formation du jet atomique.

unité de volume dans le four, ce nombre dans le jet au point $\mathrm{A}$ est sensiblement $n(\mathrm{~A}) \# n_{0} \Omega$. Donc d'une manière générale à l'abscisse $z$ :

$$
n(z)=n(h) \frac{h^{2}}{z^{2}} \text {. }
$$

La divergence du jet est la cause d'un effet Doppler résiduel caractérisé dans l'observation suivant $O y$ par sa largeur spectrale relative :

$$
\frac{\Delta \lambda}{\lambda}=v_{0} \frac{s}{2 h c}
$$

où $c$ est la vitesse de la lumière, et $v_{0}=\sqrt{3 \mathrm{kT} / \mathrm{m}}$ est la vitesse quadratique moyenne des atomes dans le four en même temps d'ailleurs que la vitesse la plus probable dans le jet.

Pour notre montage, on trouve que $\Delta \lambda / \lambda \# 10^{-7}$, valeur confirmée par des mesures de largeur de raies d'émission (Vedel) [9].

\section{Le profil spectral d'absorption du jet et son excita-} tion. - Dans un volume donné, l'absorption monochromatique est proportionnelle au nombre d'atomes absorbant la fréquence de la bande $v$ à $v+\mathrm{d} v$ considérée. Soit $\mathrm{d} n(\mathrm{~A}, v)=\mathrm{d}^{2} N_{\mathrm{v}} / \mathrm{d} V$ la densité de ces atomes entourant le point $\mathrm{A}$.
Pour établir le profil d'absorption du jet dans un montage déterminé, il suffit, si le coefficient volumique d'absorption $\alpha(v, \mathrm{~A})$ est connu, d'intégrer l'équation de transfert du rayonnement sur les fréquences et sur l'espace parcouru. Le résultat dépend donc fortement de la direction suivant laquelle le jet est éclairé, ceci pour les raisons suivantes :

- la profondeur géométrique du jet dépend de la direction,

- l'effet Doppler régit $n(v)$, comme nous allons le voir.

Dans le cas d'excitations transversales, ce profil dépend du facteur de collimation C. L'effet Doppler se traduit par un élargissement de la bande d'absorption qui tend à prendre un profil gaussien.

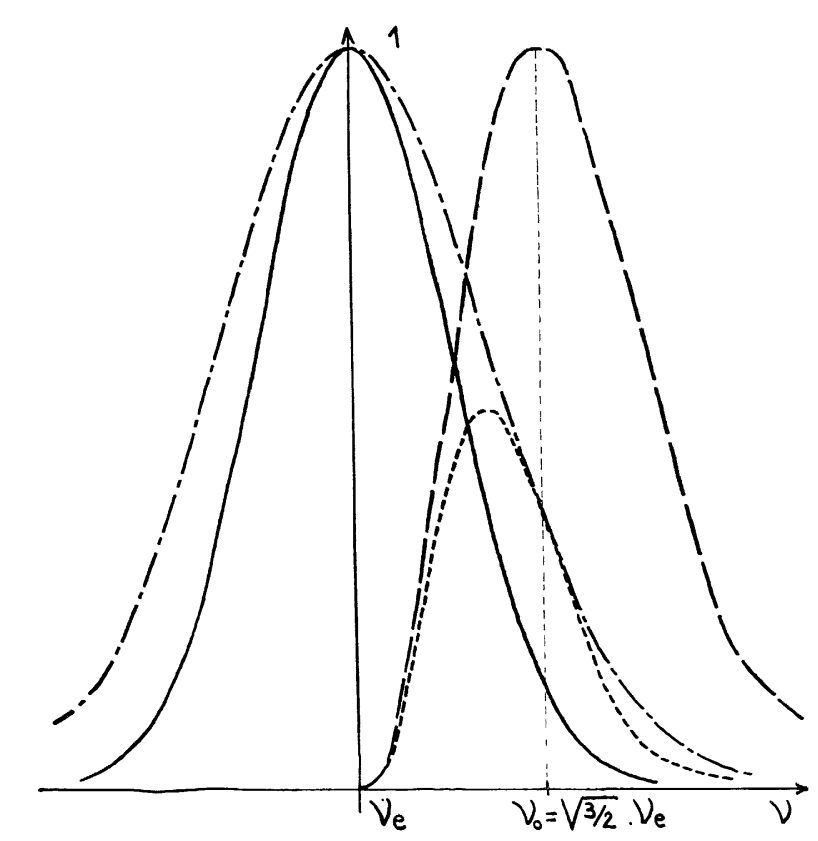

FIG. 3. - Efficacité de la photoexcitation axiale.

$G(v)$ profil spectral de l'éclairement excitateur.
$\ldots \ldots \ldots(v)$ profil spectral de sa bande d'absorption dans le jet. $G^{2}(v)$.

-.... $G(v) . J(v)$.

Très étroite dans l'épaisseur du jet (suivant $O y$ ), cette bande s'élargit considérablement dans la direction perpendiculaire, c'est-à-dire suivant la tranche du jet (direction $O x$ ) (Fig. 1). C'est donc ainsi qu'il faudrait l'exciter si l'on ne disposait que de sources conventionnelles (cathodes creuses, par exemple). On bénéficierait ainsi d'une profondeur optique importante et d'une largeur de la bande d'absorption maximale qui permettraient une meilleure interaction entre le faisceau lumineux et le jet. D'autre part, on disposerait d'une grande surface de réémission, les raies que l'on observerait suivant $O y$ ayant alors un profil très fin. Les réalisations rencontrées jusqu'à présent utilisent presque toutes un mode d'excitation transversal (Odintsov) [6] (Hinnov-Ohlendorf) [3]. 
Supposons maintenant que le rayonnement excitateur ait même direction et même sens de propagation que le jet. Le profil spectral d'une raie correspondant à la transition $h v_{\mathrm{e}}=W_{\mathrm{i}^{\prime}}-W_{\mathrm{i}}$ émise au niveau du four est gaussien. Ce profil centré sur la fréquence $v_{\mathrm{e}}$ est plus ou moins altéré ensuite pendant le parcours $\mathrm{du}$ rayonnement dans la vapeur jusqu'au point $\mathrm{A}$. Soit $\mathrm{d} E v=E\left(\mathrm{~A}, v-v_{\mathrm{e}}\right) \mathrm{d} v$ l'éclairement excitateur en $\mathrm{A}$ pour la bande $v, v+\mathrm{d} v$. Le nombre d'atomes par unité de volume en $\mathrm{A}$ ayant une vitesse $v, v+\mathrm{d} v$ est :

$$
\mathrm{d} n_{v}=\frac{3 \sqrt{6}}{\sqrt{\pi}} n(\mathrm{~A}) \frac{v^{2}}{v_{0}^{3}} \exp \left(-\frac{3}{2} \frac{v^{2}}{v_{0}^{2}}\right) \mathrm{d} v .
$$

Pour les $\mathrm{d} n_{\mathrm{i}, v}=r_{\mathrm{i}} \mathrm{d} n_{v}$ d'entre eux qui sont au niveau $W_{\mathrm{i}}$, l'excitation vers le niveau $W_{\mathrm{i}}$, est possible si leur vitesse

$$
v=c \frac{v-v_{\mathrm{e}}}{v_{\mathrm{e}}} .
$$

Le nombre d'atomes qui sera excité par unité de volume dans la bande $v, v+\mathrm{d} v$ est donc :

$$
\begin{aligned}
& \mathrm{d} n_{\mathrm{i}}(\mathrm{A}, v)=\frac{3 \sqrt{6}}{\sqrt{\pi}} r_{\mathrm{i}} \bar{\omega}_{\mathrm{i}} n(\mathrm{~A}) \frac{v-v_{\mathrm{e}}}{\left(v_{0}-v_{\mathrm{e}}\right)^{3}} \times \\
& \times \exp \left(-\frac{3}{2} \frac{\left(v-v_{\mathrm{e}}\right)}{\left(v_{0}-v_{\mathrm{e}}\right)^{2}}\right) \mathrm{d}\left(v-v_{\mathrm{e}}\right)
\end{aligned}
$$

où $\bar{\omega}_{\mathrm{i}}$ représente la probabilité de transition du niveau (i) au niveau (i').

Dans ces conditions, l'énergie absorbée par unité de volume est :

$w_{\mathrm{abs}}\left(\mathrm{A}, v_{\mathrm{e}}\right)=\frac{h v_{\mathrm{e}} \bar{\omega}_{\mathrm{i}}}{E_{\max }\left(\mathrm{A}, v_{\mathrm{e}}\right)} \int_{0}^{\infty} E\left(\mathrm{~A}, v-v_{\mathrm{e}}\right) \cdot \mathrm{d} n_{\mathrm{i}}\left(v-v_{\mathrm{e}}\right)$

pour une densité volumique d'énergie incidente :

$$
u\left(A, v_{\mathrm{e}}\right)=\int_{-\infty}^{+\infty} \frac{4 \pi}{\Omega} \frac{E\left(A, v-v_{\mathrm{e}}\right) \mathrm{d}\left(v-v_{\mathrm{e}}\right)}{c} .
$$

On peut caractériser l'efficacité de l'excitation par le rapport

$$
e\left(\mathrm{~A}, v_{\mathrm{e}}\right)=\frac{w_{\mathrm{abs}}\left(\mathrm{A}, v_{\mathrm{e}}\right)}{u\left(\mathrm{~A}, v_{\mathrm{e}}\right)} .
$$

Si on admet pour $E\left(\mathrm{~A}, v-v_{\mathrm{e}}\right)$ un profil gaussien de la forme

$$
E_{\mathrm{G}}\left(\mathrm{A}, v-v_{\mathrm{e}}\right)=E_{\mathrm{Gmax}}\left(v_{\mathrm{e}}\right) \cdot \exp -\frac{3}{2} \frac{\left(v-v_{\mathrm{e}}\right)^{2}}{\left(v_{0}-v_{\mathrm{e}}\right)^{2}} .
$$

On trouve que

$$
e_{\mathrm{G}}\left(\mathrm{A}, v_{\mathrm{e}}\right)=\frac{\sqrt{3}}{4 \sqrt{\pi}} \frac{\bar{\omega}_{\mathrm{i}} r_{\mathrm{i}} h c v_{\mathrm{e}} n(\mathrm{~A})}{E_{\mathrm{Gmax}}\left(v_{\mathrm{e}}\right) \cdot\left(v_{0}-v_{\mathrm{e}}\right) \Omega} .
$$

Si on remarque que l'éclairement total de cette raie est

$$
\begin{aligned}
E_{\mathrm{G}, v_{\mathrm{e}}}(\mathrm{A}) & =2 \int_{0}^{\infty} E_{\mathrm{G}}\left(\mathrm{A}, v-v_{\mathrm{e}}\right) \mathrm{d}\left(v-v_{\mathrm{e}}\right) \\
& =2 \frac{\pi}{6} E_{\mathrm{G} \max }(\mathrm{A}) \cdot\left(v_{0}-v_{\mathrm{e}}\right)
\end{aligned}
$$

et que d'autre part la quantité

$$
n_{h v_{\mathrm{e}}}(\mathrm{A})=\frac{E_{\mathrm{G}, v_{\mathrm{e}}}(\mathrm{A})}{h c v_{\mathrm{e}}}
$$

représente le nombre de photons $h v_{\mathrm{e}}$ par unité de volume correspondant à cet éclairement, on peut écrire que

$$
e_{\mathrm{G}}\left(\mathrm{A}, v_{\mathrm{e}}\right)=\frac{\Omega}{4 \pi} \frac{\bar{\omega}_{\mathrm{i}} \cdot n_{\mathrm{i}}(\mathrm{A})}{n_{h v_{\mathrm{e}}}(\mathrm{A})} .
$$

Cette efficacité est le quart de celle qui correspondrait au même profil spectral gaussien centré sur la même fréquence pour l'excitation et pour l'absorption.

Remarquons enfin que la perte d'éclairement dans le parcours $\mathrm{d} z$ pour la bande $v$ à $v+\mathrm{d} v$ est

$$
\begin{aligned}
\mathrm{d} E_{v} & =-w_{\mathrm{abs}}\left(\mathrm{A}, v-v_{\mathrm{e}}\right) \mathrm{d} v \mathrm{~d} z \\
& =-E\left(\mathrm{~A}, v-v_{\mathrm{e}}\right) \cdot \alpha_{\mathrm{i}}\left(\mathrm{A}, v-v_{\mathrm{e}}\right) \mathrm{d} v \mathrm{~d} z
\end{aligned}
$$

donc

$$
\alpha_{\mathrm{i}}\left(\mathrm{A}, v-v_{\mathrm{e}}\right)=\frac{h v_{\mathrm{e}} \sigma_{\mathrm{i}}}{E_{\max }\left(\mathrm{A}, v_{\mathrm{e}}\right)} \cdot \mathrm{d} n_{\mathrm{i}}(\mathrm{A}, v)
$$

2. Réalisation expérimentale. - 2.1 JET ATOMIQUE. - Celui que nous utilisons a été étudié en détail par G. Montixi (Montixi) [5] (Fig. 4).

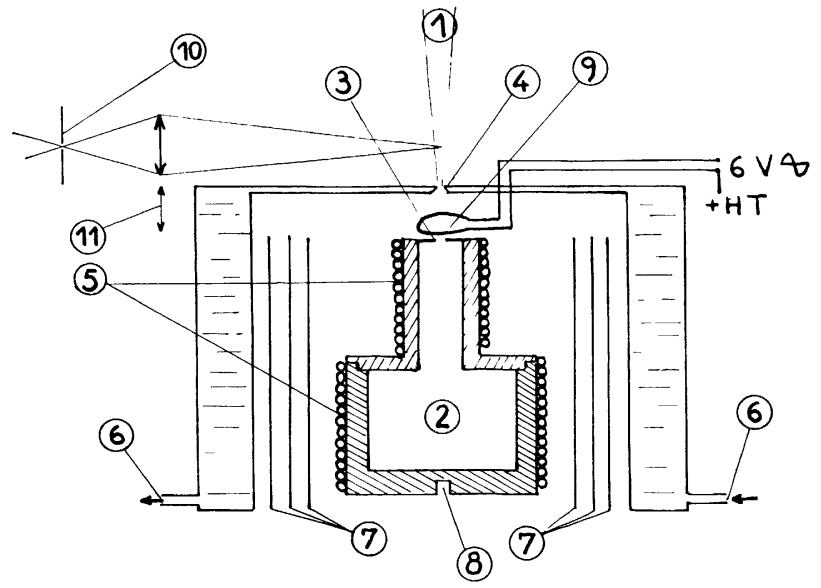

Fig. 4. - Dispositif expérimental : 1. jet atomique ; 2. four : 3 . fente inférieure ; 4. fente supérieure ; 5 . chauffage ; 6 . circulation d'eau ; 7. écrans thermiques ; 8. prise de température ; 9. anode ; 10. fente d'entrée du spectromètre; 11 . déplacement micrométrique de la lentille.

Le four contient à sa partie inférieure le produit à évaporer. La partie supérieure est démontable pour permettre le remplissage. Il est chauffé par un enroulement de thermocoax, et la température est mesurée à la base au moyen d'un thermocouple. Le four est enveloppé de trois écrans thermiques, et d'une enceinte refroidie à l'eau sur laquelle est fixée la fente supérieure.

2.2 Système D'EXCITATION OPTIQUE. - La pression qui règne dans l'espace interfente, de l'ordre de $5 \times 10^{-2}$ torr à $10^{-1}$ torr est suffisante pour permettre le maintien d'une décharge en cathode creuse. 
Dans ce but, nous y avons placé un anneau isolé, centré sur l'axe $O z$ des fentes. Il joue le rôle d'anode, la cathode étant constituée par la fente de sortie du four (Fig. 4).

La pression varie très rapidement avec la température. Si elle est trop faible, elle ne permet pas le maintien de la décharge ; si elle est trop forte, la décharge se délocalise, envahit l'intérieur du four, et le milieu ionisé participe à la formation du jet. Ceci se traduit, dans le spectre de fluorescence, par l'apparition des raies de résonance $4554 \AA$ et $4935 \AA$ de Ba II.

Un régime stable de la décharge étant indispensable, la température a été régulée très soigneusement $\left( \pm 0,5^{\circ} \mathrm{C}\right)$.

Cependant, l'évaporation du baryum issu du four provoque un dépôt sur l'anode, ce qui met systématiquement le dispositif en court-circuit au bout de quelques heures de fonctionnement. Il faut chauffer le fil d'anode entre deux expériences pour vaporiser ce dépôt.

2.3 DÉTECTION. - Elle utilise un spectromètre et un photomultiplicateur EMI $6256 \mathrm{~S}$ associé à une électronique adaptée aux faibles flux (Fig. 5).
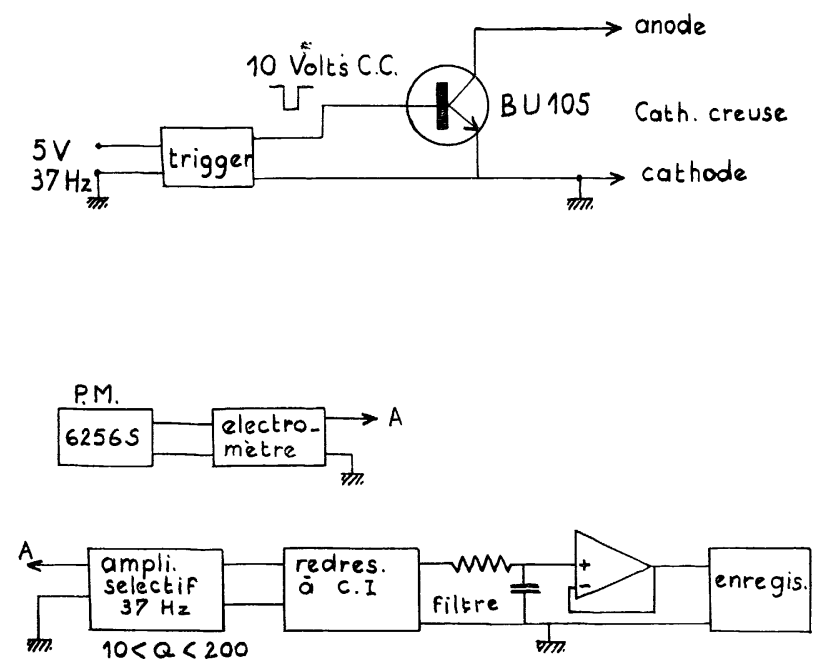

FIG. 5. - Chopper électronique et dispositif de détection.

Le spectromètre est du type Czerny-Turner, de $60 \mathrm{~cm}$ de focale, et ouvert à $f / 6$. Le réseau a 1200 traits au mm. Une lentille de quartz forme l'image du jet luminescent sur la fente d'entrée du spectromètre.

Cette fente est perpendiculaire au jet, ce qui lui permet de jouer le rôle de diaphragme explorateur lorsqu'on désire analyser le spectre de fluorescence à différentes distances de la fente de sortie du jet (donc suivant $\mathrm{Oz}$ ).

Afin d'améliorer la sensibilité de cette détection, nous avons été amenés à réaliser un système électronique, décrit ailleurs (Aubert-Baldy) [1], utilisant un interrupteur électronique haute tension sur l'alimentation de la cathode creuse. Il hache le flux lumineux excitateur à une fréquence fixe, $F_{0}=37 \mathrm{~Hz}$ (Fig. 5).
Le signal issu du photomultiplicateur passe par un amplificateur sélectif à bande étroite, centré sur $F_{0}$, dont le facteur de qualité peut être ajusté entre 10 et 100 .

Le signal alternatif, amplifié et débarrassé du bruit est ensuite redressé par un montage à circuit intégré permettant la détection de signaux supérieurs à $100 \mu \mathrm{V}$. Après filtrage, puis abaissement de l'impédance de sortie à $500 \Omega$, le signal est enregistré. Ce système permet :

- de s'affranchir des diffusions parasites (rayonnement thermique et fonds continus) qui ne sont pas modulés à $F_{0}$;

- d'améliorer le rapport signal/bruit, la bande passante de bruit pouvant être réduite jusqu'à $0,5 \mathrm{~Hz}$.

3. Mesures. - 3.1 Mesures PrÉliminaires. Cette technique d'excitation étant nouvelle, nous avons entrepris une étude systématique des paramètres agissant sur le spectre de fluorescence.

3.1 .1 Spectre émis par la cathode creuse (25 mA $<\mathrm{I}<80 \mathrm{~mA})$. - Les enregistrements montrent la présence de presque toutes les raies du Ba I, et des raies principales du $\mathrm{Ba}$ II. Nous avons reproduit le diagramme des transitions du Ba I observées dans la cathode creuse (Russel-Moore) [7] (Fig. 6).

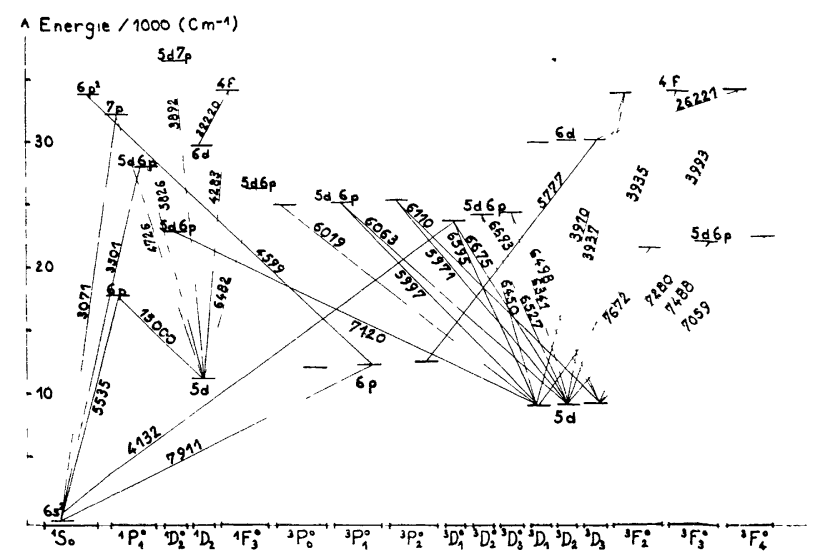

FIG. 6. - Principales transitions observées dans le spectre de fluorescence.

On peut y noter en particulier la forte intensité des raies d'intercombinaison $4599 \AA\left(6 \mathrm{p}^{2}{ }^{1} \mathrm{~S}_{0}-6 \mathrm{~s} 6 \mathrm{p}^{3} \mathrm{P}_{1}\right)$ $4132 \AA\left(5 \mathrm{~d} 6 \mathrm{p}{ }^{3} \mathrm{D}_{1}-6 \mathrm{~s}^{2}{ }^{1} \mathrm{~S}_{0}\right), 7911 \AA\left(6 \mathrm{~s} 6 \mathrm{p}^{3} \mathrm{P}_{1}-6 \mathrm{~s}^{2}{ }^{1} \mathrm{~S}_{0}\right)$ qui contribuent à peupler les niveaux triplets et en particulier les niveaux métastables $5 \mathrm{~d}^{3} \mathrm{D}_{1,2,3}$ dans le jet.

3.1.2 Spectre de fluorescence du jet. - Les raies enregistrées sont environ 1000 fois plus faibles que leurs homologues du spectre de cathode creuse. Notons que les seules transitions observées n'aboutissent qu'au niveau fondamental, ou aux niveaux métastables (Fig. 6).

Mise à part la raie de résonance, $\lambda=5535 \AA$ qui est environ 100 fois plus forte que les autres, les raies des 
singulets les plus intenses sont les transitions $4726 \AA$ (6s $7 \mathrm{p}^{1} \mathrm{P}_{1}^{0}$-6s $\left.5 \mathrm{~d}^{1} \mathrm{D}_{2}\right)$ et $5826 \AA\left(5 \mathrm{~d} 6 \mathrm{p}^{1} \mathrm{P}_{1}^{0}-6 \mathrm{~s} 5 \mathrm{~d}^{1} \mathrm{D}_{2}\right)$. Le retour au niveau fondamental s'effectue par les transitions $3071 \AA$ et $3501 \AA$. La raie $4283 \AA$ (6s $4 \mathrm{f}^{1} \mathrm{~F}_{3}^{0}-6 \mathrm{~s} 5 \mathrm{~d}^{1} \mathrm{D}_{2}$ ) est particulièrement renforcée. L'intensité de cette raie, relativement plus forte dans le spectre de fluorescence du jet que dans le spectre excitateur prouve que l'excitation photonique enrichit notablement le jet en atomes métastables $5 \mathrm{~d}^{1} \mathrm{D}_{2}$.

En effet, l'excitation photonique des atomes au niveau $6 \mathrm{~s} 4 \mathrm{f}^{1} \mathrm{~F}_{3}^{0}$ ne peut se faire pratiquement que par l'absorption de la raie $4283 \AA$ par un atome métastable. La transition spontanée la plus probable à partir du niveau $6 \mathrm{~s} 4 \mathrm{f}^{1} \mathrm{~F}_{3}^{0}$ est le retour au niveau métastable avec réémission de la même raie dans le spectre de fluorescence. On peut négliger, en première approximation, le dépeuplement du niveau $6 \mathrm{~s} 4 \mathrm{f}^{1} \mathrm{~F}_{3}^{0}$ dû à la transition $22220 \AA$ (6s $\left.4 \mathrm{f}^{1} \mathrm{~F}_{3}^{0}-6 \mathrm{~s} 6 \mathrm{~d}^{1} \mathrm{D}_{2}\right)$. L'observation de la raie $4283 \AA$ renseigne donc directement sur l'évolution de la concentration en atomes métastables singulets du jet si les conditions de cette observation conservent une intensité locale d'excitation fixe, ou si celle-ci est contrôlée.

Les raies homologues des séries de triplets apparaissent aussi, mais avec des intensités plus faibles. En particulier, la raie $3993 \AA$ (6s $4 \mathrm{f}^{3} \mathrm{~F}_{4}^{0}-6 \mathrm{~s} 5 \mathrm{~d}{ }^{3} \mathrm{D}_{3}$ ) est relativement renforcée elle aussi. Ces atomes métastables sont vraisemblablement produits en partie par pompage optique dans le jet, mais aussi par la décharge cathodique en amont du jet.

Ces observations sont à rapprocher de celles qui ont été faites sur l'excitation du spectre d'émission de jets de strontium par électrons lents (Chantrel-MontixiVedel) [2]. Le spectre des triplets n'existait pas, et les transitions ${ }^{1} \mathrm{~F}^{0}{ }_{-1}^{1} \mathrm{D}$ étaient renforcées.

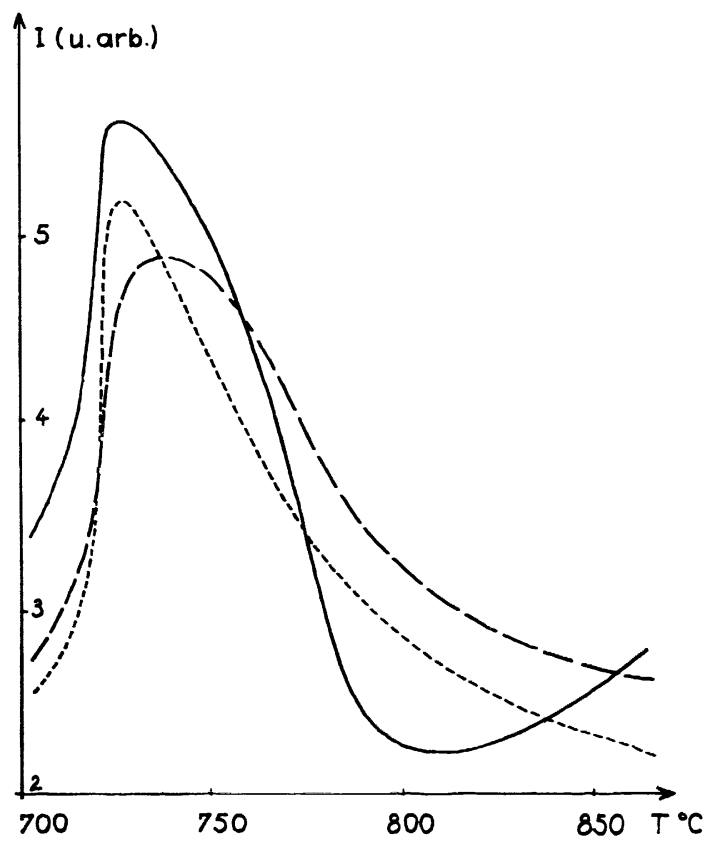

(a)
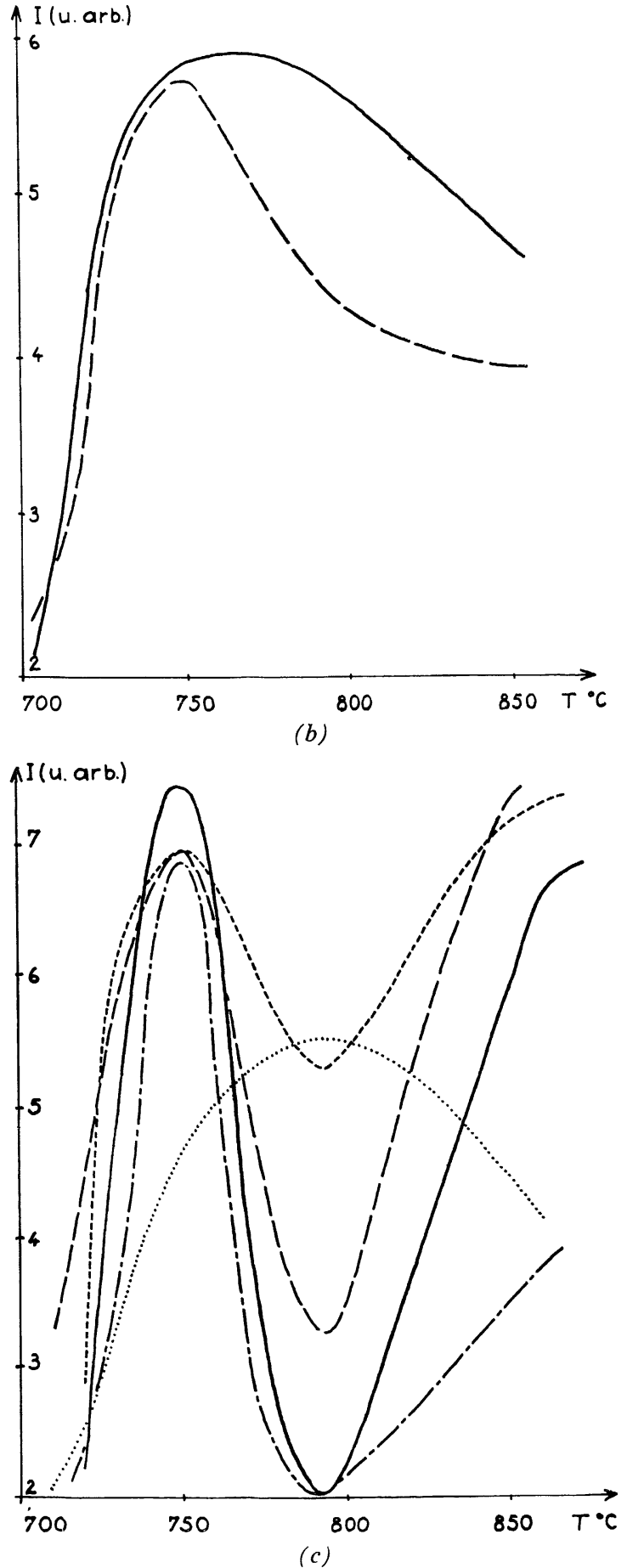

Fig. 7. - Intensité des raies de fluorescence en fonction de la température du four :

a) $3071 \AA\left(6 \mathrm{~s} 7 \mathrm{p}{ }^{1} \mathrm{P}_{1}^{0}-6 \mathrm{~s}^{2}{ }^{1} \mathrm{~S}_{0}\right)$ $5535 \AA\left(6 \mathrm{~s} 6 \mathrm{p}^{1} \mathrm{P}_{1}^{0}-6 \mathrm{~s}^{2}{ }^{1} \mathrm{~S}_{0}\right)---$

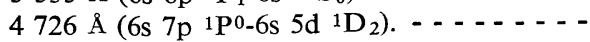

b) $5826 \AA\left(5 \mathrm{~d} 6 \mathrm{p}^{1} \mathrm{P}_{1}^{0}-6 \mathrm{~s} 5 \mathrm{~d}{ }^{1} \mathrm{D}_{2}\right)$ $4283 \AA\left(6 \mathrm{~s} 4 \mathrm{f}^{1} \mathrm{~F}_{3}^{0}-6 \mathrm{~s} 5 \mathrm{~d}^{1} \mathrm{D}_{2}\right)$.

c) $6595 \AA\left(5 \mathrm{~d} 6 \mathrm{p}^{3} \mathrm{D}_{1}^{0}-6 \mathrm{~s} 5 \mathrm{~d}^{3} \mathrm{D}_{1}\right)$ $6675 \AA\left(5 \mathrm{~d} 6 \mathrm{p}^{3} \mathrm{D}_{1-6 \mathrm{~s}}^{0} 5 \mathrm{~d}^{3} \mathrm{D}_{2}\right)$ $6693 \AA\left(5 \mathrm{~d} 6 \mathrm{p}^{3} \mathrm{D}_{2}^{0}-6 \mathrm{~s} 5 \mathrm{~d}^{3} \mathrm{D}_{3}\right)$ $4132 \AA\left(5 \mathrm{~d} 6 \mathrm{p}^{3} \mathrm{D}_{1}^{0}-6 \mathrm{~s}^{2}{ }^{1} \mathrm{~S}_{0}\right)-\ldots-\ldots$ $3993 \AA\left(6 s 4 \mathrm{f}^{3} \mathrm{~F}_{4}^{0}-6 \mathrm{~s} 5 \mathrm{~d}^{3} \mathrm{D}_{3}\right) . \ldots . . . .$. 
3.1.3 Influence de la température du four. - Nous avons tracé les courbes décrivant l'influence de la température sur l'intensité des raies observées. Un certain nombre de remarques nous ont conduits à les classer en trois groupes (Fig. 7a, $b, c$ ).

En $a$ ), nous trouvons des raies présentant un maximum d'intensité vers $730^{\circ} \mathrm{C}\left(2 \times 10^{-1}\right.$ torr $)$. Au-delà de cette température, les courbes décroissent par suite de l'absorption de la raie $3071 \AA$ dans la cathode creuse. On peut ainsi noter l'identité parfaite des courbes qui décrivent le comportement des raies $4726 \AA\left(6 \mathrm{~s} 7 \mathrm{p}{ }^{1} \mathrm{P}_{1}^{0} 6 \mathrm{~s} 5 \mathrm{~d}^{1} \mathrm{D}_{2}\right)$ et $3071 \AA\left(6 \mathrm{~s} 7 \mathrm{p}{ }^{1} \mathrm{P}_{1^{-}}^{0}\right.$ $6 \mathrm{~s}^{2}{ }^{1} \mathrm{~S}_{0}$ ) issues du même niveau $6 \mathrm{~s} 7 \mathrm{p}{ }^{1} \mathrm{P}_{1}^{0}$.

En $b$ ), nous trouvons deux raies dont le maximum d'intensité s'est déplacé vers $750^{\circ} \mathrm{C}\left(3 \times 10^{-1}\right.$ torr $)$ : la raie $4283 \AA\left(6 \mathrm{~s} 4 \mathrm{f}^{1} \mathrm{~F}_{3} 6 \mathrm{~s} 5 \mathrm{~d}^{1} \mathrm{D}_{2}\right)$ dont on a déjà parlé et la raie $5826 \AA\left(5 \mathrm{~d} 6 \mathrm{p}^{1} \mathrm{P}_{1} 6 \mathrm{~s} 5 \mathrm{~d}^{1} \mathrm{D}_{2}\right)$ qui résulte d'une transition entre le niveau de double excitation $5 \mathrm{~d} 6 \mathrm{p}^{1} \mathrm{P}_{1}$ et le niveau métastable singulet. Ces deux raies proviennent donc de la photoexcitation d'atomes métastables singulets avec réémission aux mêmes longueurs d'onde par transition inverse. Comme, d'autre part, on n'a pas observé d'évolution notable de la décharge excitatrice avec la température il y a tout lieu de croire qu'à $750^{\circ} \mathrm{C}$, le maximum d'intensité de la fluorescence pour ces deux raies correspond aussi à la concentration maximale en atomes singulets métastables dans le jet. Il serait intéressant de le confirmer en détectant les atomes métastables par d'autres procédés, mais nous ne sommes pas équipés pour le faire.

En c), l'allure des courbes est plus compliquée. Notons que le minimum d'intensité de différentes transitions dépeuplant les niveaux métastables $5 \mathrm{~d}^{3} \mathrm{D}_{1,2,3}$ coïncide avec un maximum pour la raie $3993 \AA$ (6s $\left.4 \mathrm{f}^{3} \mathrm{~F}_{4}^{0}-6 \mathrm{~s} 5 \mathrm{~d}^{3} \mathrm{D}_{3}\right)$ dont l'intensité est proportionnelle au nombre d'atomes excités dans l'état métastable $5 \mathrm{~d}^{3} \mathrm{D}_{3}$. Remarquons que la raie d'intercombinaison $4132 \AA$ doit jouer un rôle certain dans le mécanisme de dépopulation des niveaux $5 \mathrm{~d}^{3} \mathrm{D}_{1,2,3}$.

3.1.4 Influence de l'intensité de cathode. - Nous avons fait varier l'intensité entre $25 \mathrm{~mA}$ et $100 \mathrm{~mA}$ (Fig. 8). Toutes les courbes décroissent d'abord et présentent un minimum vers $35 \mathrm{~mA}$. La raie de résonance voit son minimum déplacé à $50 \mathrm{~mA}$.

3.2 DéCROISSANCE DES RAIES EN FONCTION DE LA DISTANCE $z$. - La fente d'entrée du spectromètre isole une tranche de l'image du jet d'épaisseur $\mathrm{d} z$. La profondeur géométrique du jet, à la hauteur $z$, est égale à $y=z / \mathcal{C}$ où $\mathcal{C}$ est le facteur de collimation du jet.

Les mesures effectuées sur différentes raies ont permis de relever deux types de courbes :

3.2.1 Décroissance de la raie de résonance (5 $535 \AA$ ) (Fig. 9a). - En coordonnées semi-logarithmiques, la décroissance observée est hyperbolique (Fig. 9a). Compte tenu de la précision des mesures, il apparaît

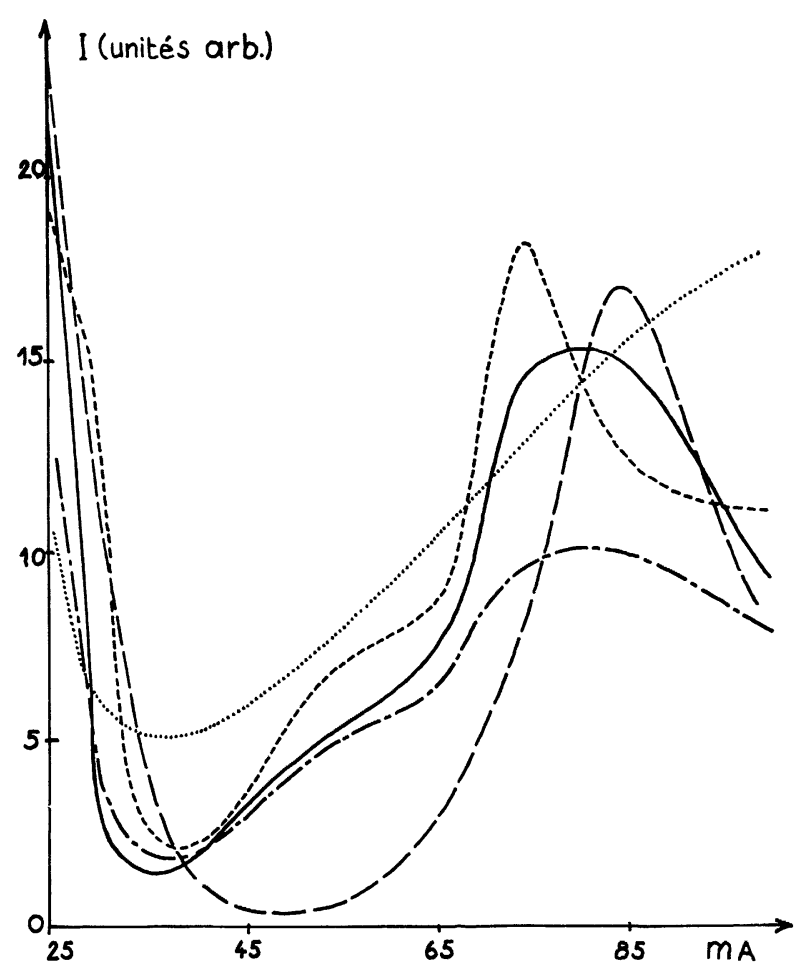

FIG. 8. - Intensité des raies de fluorescence en fonction de l'intensité du courant de cathode creuse en mA. $5826 \AA, 5535 \AA$, $3071 \AA, 4726 \AA, 3993 \AA$.

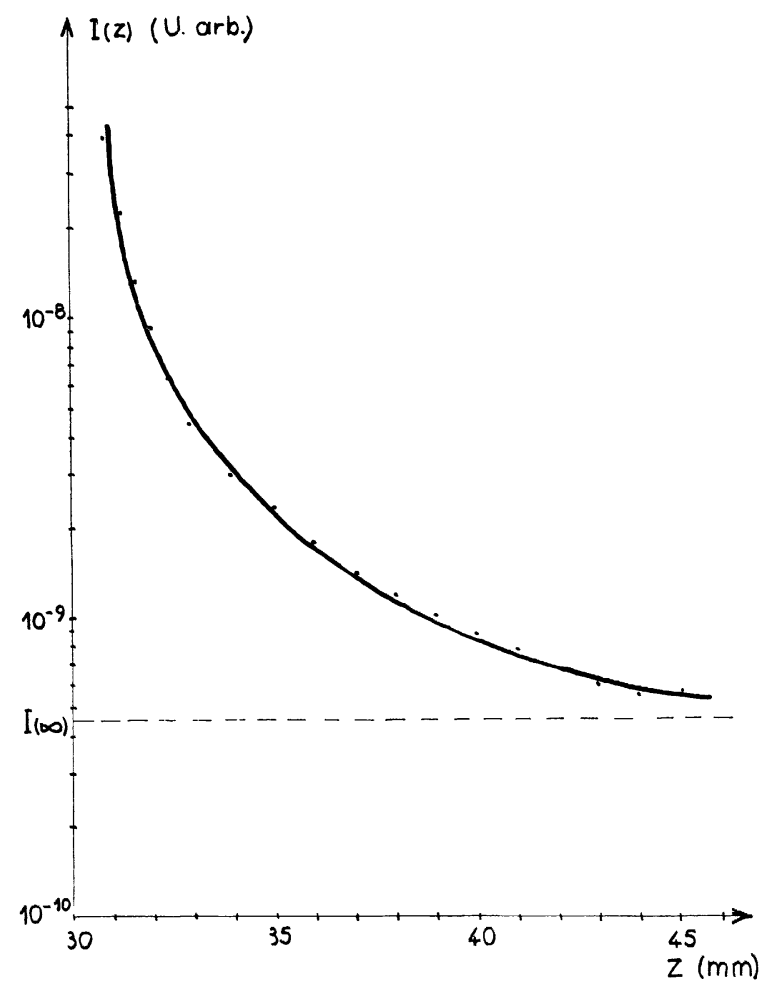

Fig. 9. - Décroissance de l'intensité de la raie de résonance $5535 \AA$ avec la hauteur $z$.

que l'intensité $I(z)$ tend vers une limite non nulle, $I(\infty)$, quand la hauteur $z$ est suffisamment grande.

Ceci se traduit sur le graphe par la présence d'une asymptote horizontale, vraisemblablement due à la 
réabsorption de la raie de résonance émise en fluorescence en un autre point du jet.

\subsubsection{Décroissance de l'intensité des autres raies} (Fig. 9b). - Les lois expérimentales obtenues sont du type $I(z)=I(h) \exp -\beta(\lambda) z$ (Fig. $9 b)$. En particulier la variation de l'intensité des raies de transition D-F traduit l'évolution suivant $z$ des populations des atomes dans les états métastables $5 \mathrm{~d}{ }^{1} \mathrm{D}_{2}$ et $5 \mathrm{~d}^{3} \mathrm{D}_{1,2,3}$.

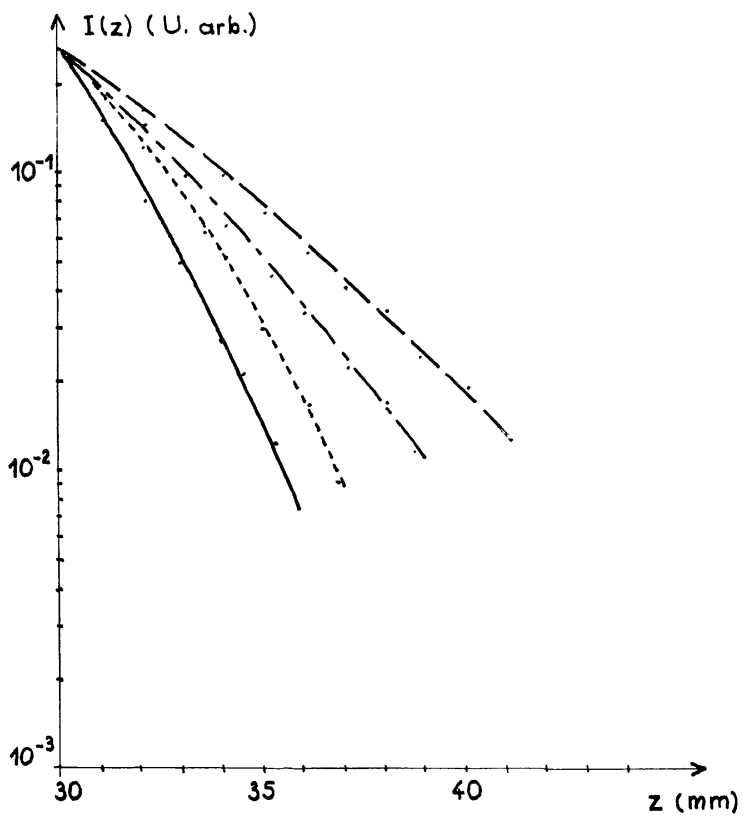

Fig. 10. - Décroissance de l'intensité de quelques raies de fluorescence avec la hauteur $z$ :

$4283 \AA\left(6 \mathrm{~s} 4 \mathrm{f}^{1} \mathrm{~F}_{3}^{0}-6 \mathrm{~s} 5 \mathrm{~d}^{1} \mathrm{D}_{2}\right)---$ $3993 \AA\left(6 s 4 \mathrm{f}^{3} \mathrm{~F}_{4}^{0}-6 \mathrm{~s} 5 \mathrm{~d}^{3} \mathrm{D}_{3}\right) \ldots \ldots$ $3071 \AA\left(6 \mathrm{~s} 7 \mathrm{p}{ }^{1} \mathrm{P}_{1}^{0}-6 \mathrm{~s}^{2}{ }^{1} \mathrm{~S}_{0}\right) \ldots . . . . . . .$. $4726 \AA$ (6s $\left.7 \mathrm{p}{ }^{1} \mathbf{P}_{1}^{0}-6 \mathrm{~s} 5 \mathrm{~d}{ }^{1} \mathrm{D}_{2}\right)$.

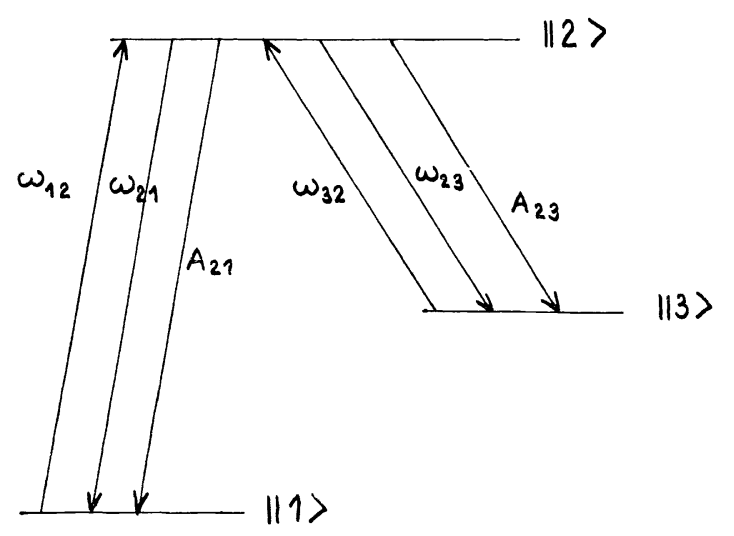

FIG. 11. - Pompage sur un modèle à trois niveaux.

4. Application à la détermination des durées de vie des états excités. - L'état du jet, à la hauteur $z$ comptée à partir de la fente supérieure peut être décrit par les populations $N_{n}(z)$ des divers états $n$ à cet endroit. L'évolution de ces populations pendant le temps $\mathrm{d} t$ est régie par la relation :

$$
\begin{aligned}
\dot{N}_{n}(z, t)=\sum_{n^{\prime}} A_{n^{\prime} n} N_{n^{\prime}}(z, t) & -A_{n n^{\prime}} N_{n}(z, t)+ \\
+ & \bar{\omega}_{n^{\prime} n} N_{n^{\prime}}(z, t)-\bar{\omega}_{n n^{\prime}} N_{n}(z, t)
\end{aligned}
$$

où $\dot{N}_{n}=\mathrm{d} N_{n} / \mathrm{d} t$ et où $A_{n^{\prime} n}$ et $A_{n n^{\prime}}$ représentent respectivement les probabilités de relaxation du niveau $\| n^{\prime}>$ vers le niveau $\| n>$ et de $\| n>$ vers $\| n^{\prime}>$. $\bar{\omega}_{n n^{\prime}}$ et $\bar{\omega}_{n^{\prime} n}$ sont les probabilités de transition par pompage de $\| n>$ vers $\| n^{\prime}>$ et de $\| n^{\prime}>$ vers $\| n>$. (Bertein) [12]. Elles sont reliées aux coefficients d'Einstein par la relation:

$$
\bar{\omega}_{n n^{\prime}}=U\left(v_{n n^{\prime}}, \quad T_{n n^{\prime}} z\right) \times B_{n^{\prime} n}
$$

où $U\left(v_{n n^{\prime}}, T_{n n^{\prime}} z\right)$ est la densité d'énergie de pompage, qui est une fonction de la température fictive de pompage $T_{n n^{\prime}}$ et de $v_{n n^{\prime}}$, fréquence de la transition $\|n>\rightarrow\| n^{\prime}>$.

Rappelons que la température de pompage $T_{n n^{\prime}}$ est celle du rayonnement thermique, qui, appliquée au gaz assurerait un pompage équivalent, c'est-à-dire une probabilité d'absorption égale à $\bar{\omega}_{n n^{\prime}}$.

Entre les coefficients d'Einstein $A$ et $B$, on a les relations connues :

$$
A_{n n^{\prime}}=\frac{8 \pi h v_{n n^{\prime}}^{3}}{c^{3}} B_{n n^{\prime}}=\frac{8 \pi h v_{n n^{\prime}}^{3}}{c^{3}} \frac{G_{n^{\prime}}}{G_{n}} B_{n^{\prime} n}
$$

$G_{n^{\prime}}$ et $G_{n}$ étant respectivement les poids statistiques des niveaux $\| n^{\prime}>$ et $\| n>$.

La détermination des grandeurs $N_{n}$ et $U\left(v_{n n^{\prime}}, T_{n n^{\prime}} z\right)$ permet d'atteindre les forces d'oscillateur $f_{n n^{\prime}}$ et les durées de vie des états excités. Montrons-le dans le cas simple d'un modèle à trois niveaux $\|1>\| 2>$,, $\| 3>$; $\| 1>$ est le fondamental, $\| 2>$ le premier niveau excité, et $\| 3>$ un niveau métastable.

Dans ce modèle, on observe une relaxation de $\|2>\rightarrow\| 1>$, et de $\|2>\rightarrow\| 3>$, et le pompage s'effectue de $\|1>\rightarrow\| 2>$ et de $\|3>\rightarrow\| 2>$.

Dans le domaine optique et aux températures considérées, $A_{12}$ et $A_{32}$ sont négligeables devant $A_{21}$ et $A_{23}$. D'autre part, le régime d'équilibre étant atteint, les $\dot{N}_{n}$ sont nuls.

La résolution du système d'équations correspondant donne les populations $N_{n}$

$$
\begin{aligned}
& N_{1}(z)=N(z) \frac{\bar{\omega}_{32}\left(\bar{\omega}_{21}+A_{21}\right)}{\bar{\omega}_{12}\left(\bar{\omega}_{32}+A_{23}+\bar{\omega}_{23}\right)+\bar{\omega}_{32}\left(\bar{\omega}_{21}+A_{21}\right)} \\
& N_{2}(z)=N(z) \frac{\bar{\omega}_{12} \bar{\omega}_{32}}{\bar{\omega}_{12}\left(\bar{\omega}_{32}+A_{23}+\bar{\omega}_{23}\right)+\bar{\omega}_{32}\left(\bar{\omega}_{21}+A_{21}\right)} \\
& N_{3}(z)=N(z) \frac{\bar{\omega}_{12}\left(A_{23}+\bar{\omega}_{23}\right)}{\bar{\omega}_{12}\left(\bar{\omega}_{32}+A_{23}+\bar{\omega}_{23}\right)+\bar{\omega}_{32}\left(\bar{\omega}_{21}+A_{21}\right)}
\end{aligned}
$$

avec $N_{1}(z)+N_{2}(z)+N_{3}(z)=N(z)$, nombre total d'atomes à la hauteur $z$, les probabilités $\bar{\omega}_{n n^{\prime}}$ s'expriment en fonction des coefficients d'Einstein $A$ sous la forme :

$$
\bar{\omega}_{n n^{\prime}}=u\left(v_{n n^{\prime}}\right) A_{n^{\prime} n} \frac{\gamma}{v_{n^{\prime} n}^{3}} \quad \text { où } \quad \gamma=\frac{c^{3}}{8 \pi h} .
$$


Ceci permet d'écrire l'expression des populations :

$$
\begin{aligned}
& N_{2}(z)=\frac{N(z)}{\Delta} U\left(v_{12}\right) U\left(v_{23}\right) \\
& N_{3}(z)=\frac{N(z)}{\Delta} U\left(v_{21}\right)\left(\gamma v_{23}^{3}+U\left(v_{23}\right) \frac{G_{2}}{G_{3}}\right) \\
& N_{1}(z)=\frac{N(z)}{\Delta} U\left(v_{23}\right)\left(\gamma v_{21}^{3}+U\left(v_{21}\right) \frac{G_{1}}{G_{2}}\right)
\end{aligned}
$$

où

$$
\begin{aligned}
\Delta=U & \left(v_{21}\right) U\left(v_{23}\right) \times \\
& \times\left(1+\frac{G_{2}}{G_{3}}+\frac{G_{1}}{G_{2}}\right)+\gamma v_{23}^{3} U\left(v_{21}\right)+\gamma v_{21}^{3} U\left(v_{23}\right) .
\end{aligned}
$$

La mesure absolue des densités d'énergie de pompage à la hauteur $z$ dans le jet permet donc de calculer les populations $N_{n}(z)$ des divers états excités à cet endroit du jet.

D'autre part, les intensités $P_{n n^{\prime}}(z)$ des différentes raies de fluorescence sont reliées aux populations $N_{n}(z)$ des niveaux $\| n>$ :

$$
P_{n n^{\prime}}(z)=N_{n}(z) h v A_{n^{\prime} n} \frac{\Omega}{4 \pi},
$$

où $\Omega$ est l'angle solide sous lequel s'effectue l'observation.

Cette relation permet d'accéder aux coefficients $A_{n^{\prime} n}$ et, de là, aux forces d'oscillateur des différentes transitions puisque

$$
A_{n n^{\prime}}=\frac{2 \pi \mathrm{e}^{2}}{\varepsilon_{0} m c^{3}} v_{n n^{\prime}}^{2} \frac{G_{n^{\prime}}}{G_{n}} f_{n n^{\prime}} .
$$

La mesure des intensités $P_{n n^{\prime}}(z)$ permet donc de déterminer les $f_{n n^{\prime}}$ et les durées de vie $\tau_{n}$ des états excités, données par la relation:

$$
\tau_{n}^{-1}=\sum_{n^{\prime}} A_{n n^{\prime}}
$$

En fait, il faut considérer tous les niveaux à partir desquels on observe une transition ayant une intensité notable en fluorescence. Dans le cas du baryum, une quinzaine de niveaux interviennent, ce qui permet l'exploitation numérique des mesures.

En ce qui concerne les transitions D-F $4283 \AA$ et $6482 \AA$, le calcul montre que pour un pompage intense, le rapport des populations des deux niveaux F considérés tend en première approximation vers le rapport de leurs dégénérescences respectives. Ceci permet, connaissant le rapport des intensités de ces deux raies émises en fluorescence d'en déduire le rapport des durées de vie de ces deux états $F$. Ceci est bien sûr transposable aux transitions D-F $3993 \AA$ et $7059 \AA$.

Nous venons de voir que la détermination à la fois des densités d'énergie $u\left(v_{n n^{\prime}}, T_{n n^{\prime}}, z\right)$ et des intensités des raies de fluorescence $P_{n n^{\prime}}(z)$ à une même hauteur $z$ était nécessaire pour calculer les coefficients d'Einstein. Les mesures des densités d'énergie s'effectuent de la manière suivante : un fil diffusant est placé dans le jet à la hauteur $z$ parallèle à la fente de sortie du jet. Le spectre ainsi analysé à la hauteur $z$ se compose du spectre de fluorescence et du spectre de diffusion. Des mesures antérieures nous ayant montré que l'intensité des raies de diffusion sur le fil était environ 100 fois plus forte que celles de leurs homologues du spectre de fluorescence, nous pouvons négliger la contribution de ces dernières. La mesure faite dans ces conditions permet de connaître la densité d'énergie de rayonnement monochromatique de pompage à un facteur constant près. Ce facteur pratiquement indépendant de la longueur d'onde peut être déterminé en remplaçant le four du jet par un corps noir étalonné.

Par ailleurs la mesure absolue des $P_{n n^{\prime}}(z)$ peut s'effectuer en utilisant un filament étalonné à la hauteur $z$.

5. Conclusion. - Nous avons décrit une technique originale de photoexcitation axiale dans un jet atomique. Des mesures préliminaires nous ont permis d'optimiser les paramètres agissant sur la fluorescence, et d'en suivre l'évolution suivant la hauteur dans le jet. Le calcul nous a montré que des mesures de densités de rayonnement de pompage et d'intensités de raies de fluorescence que nous effectuons actuellement sur le baryum conduisent à la connaissance des durées de vie des états excités et des forces d'oscillateurs.

\section{Bibliographie}

[1] Aubert, D., Baldy, A., Toute l'Electronique, 1973.

[2] Chantrel, H., Montixi, G., Vedel, M., C. R. Hebd. Séan. Acad. Sci. B 264 (1967) 1651-1652.

[3] Hinnov, A. V., Ohlendorf, I. A., J. Chem. Phys. 50 (1969) 3005-3010.

[4] IshiI, I. L., Ohlendorf, I. A., Phys. Lett. 38A (1972) 119-120.

[5] Montixi, G., 1966, Thèse de Spécialité, Marseille.

[6] Odintsov, L. P., Opt. and Spectrosc. 14 (1963) 172-176.
[7] Russel, H., Moore, C., J. Res. Nat. Bur. Stand. 55 (1955) 299-306.

[8] Stanley, R., J. Opt. Soc. Am. 56 (1966) 350-356.

[9] Vedel, M., Opt. Commun. 4 (1971) 113-116.

[10] ZhiglinskiI, L. A., Kalmakov, A. G., Opt. and Spectrosc. 30 (1971) 279-281.

[11] Dickie, J. H., Kelly, O., Can. J. Phys. 49 (1971) 2630-2.

[12] Bertein, F., Base de l'électronique quantique, 1969. 